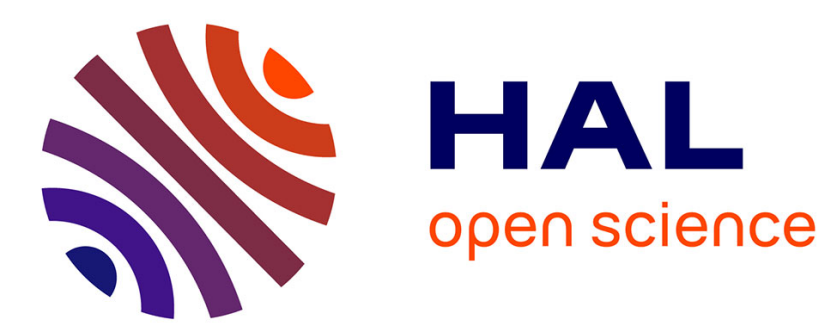

\title{
OFDM High Speed Channel Complex Gains Estimation Using Kalman Filter and QR-Detector
}

\author{
Hussein Hijazi, Laurent Ros
}

\section{To cite this version:}

Hussein Hijazi, Laurent Ros. OFDM High Speed Channel Complex Gains Estimation Using Kalman Filter and QR-Detector. ISWCS 2008 - IEEE International Symposium on Wireless Communication Systems, Oct 2008, Reykjavik, Iceland. 5 p. hal-00325329

\section{HAL Id: hal-00325329 \\ https://hal.science/hal-00325329}

Submitted on 28 Sep 2008

HAL is a multi-disciplinary open access archive for the deposit and dissemination of scientific research documents, whether they are published or not. The documents may come from teaching and research institutions in France or abroad, or from public or private research centers.
L'archive ouverte pluridisciplinaire HAL, est destinée au dépôt et à la diffusion de documents scientifiques de niveau recherche, publiés ou non, émanant des établissements d'enseignement et de recherche français ou étrangers, des laboratoires publics ou privés. 


\title{
OFDM High Speed Channel Complex Gains Estimation Using Kalman Filter and QR-Detector
}

\author{
Hussein Hijazi and Laurent Ros \\ GIPSA-lab, Department Image Signal, BP 46 - 38402 Saint Martin d'Hères - FRANCE \\ E-mail: hussein.hijazi@gipsa-lab.inpg.fr, laurent.ros@gipsa-lab.inpg.fr
}

\begin{abstract}
This paper deals with the case of a high speed mobile receiver operating in an orthogonal-frequency-divisionmultiplexing (OFDM) communication system. Assuming the knowledge of delay-related information, we propose an iterative algorithm for joint multi-path Rayleigh channel complex gains and data recovery in fast fading environments. Each complex gain time-variation, within one OFDM symbol, is approximated by a polynomial representation. Based on the Jakes process, an autoregressive (AR) model of the polynomial coefficients dynamics is built, making it possible to employ the Kalman filter estimator for the polynomial coefficients. Hence, the channel matrix is easily computed, and the data symbol is estimated with free inter-sub-carrier-interference (ICI) thanks to the use of a QRdecomposition of the channel matrix. Our claims are supported by theoretical analysis and simulation results, which are obtained considering Jakes' channels with high Doppler spreads.
\end{abstract}

Index Terms-OFDM, channel estimation, time-varying channels, Kalman filters, QR-decomposition.

\section{INTRODUCTION}

$\mathbf{O}$ RTHOGONAL frequency division multiplexing (OFDM) is an effective technique for high bit-rate transmission. In mobile communications, high speeds of terminals cause Doppler effects that could seriously affect the performance. In such case, dynamic channel estimation is needed, because the radio channel is frequency selective and time-varying, even within one OFDM symbol [4]. It is thus preferable to estimate channel by inserting pilot tones, called comb-type pilots, into each OFDM symbol [5].

For fast time-varying channel, many existing works resort to estimate the equivalent discrete-time channel taps which are modeled by a basis expansion model (BEM) [6]. The BEM methods used to model the equivalent discrete-time channel taps are Karhunen-Loeve BEM (KL-BEM), prolate spheroidal BEM (PS-BEM), complex exponential BEM (CE-BEM) and polynomial BEM (P-BEM). A great deal of attention goes to the P-BEM [7] where its modeling performance is rather sensitive to the Doppler spread though it has a better fit for low Doppler spreads than for high Doppler spreads.

As channel delay spread increases, the number of channel taps also increases, thus leading to a large number of BEM coefficients, and consequently more pilot symbols are needed. In contrast to the research described in [6], we sought to directly estimate the physical channel, instead of the equivalent discrete-time channel taps. This means estimating the physical propagation parameters such as multi-path delays and multipath complex gains. In [1], we have proposed an iterative algorithm for complex gain time-variation estimation and inter- sub-carrier-interference (ICI) suppression whose execution is done per block of OFDM symbols. This algorithm demands very high computation. In [2], we have proposed a lowcomplexity iterative algorithm based on the demonstration that each complex gain time-variation can be approximated in a polynomial fashion within several OFDM symbols. The both algorithms above reduce the ICI by using successive interference suppression (SIS), and have a good performance for normalized Doppler spread $\left(f_{d} T\right)$ up to $10 \%$.

In this paper, we present a new iterative algorithm for joint multi-path Rayleigh channel complex gains and data recovery in very fast fading environments $\left(f_{d} T>10 \%\right)$. Exploiting the channel nature, the delays are assumed invariant and perfectly estimated as we have already done in OFDM [1] [2] and CDMA [10] contexts. It should be noted that an initial, and generally accurate estimation of the number of paths and time delays can be obtained by using the MDL (minimum description length) and ESPRIT (estimation of signal parameters by rotational invariance techniques) methods [9]. However, we test by simulation the sensitivity of our algorithm to errors of estimated delays. In order to make the polynomial approximation in [2] more accurate, we approximate the time-variation of each complex gain within one OFDM symbol by a polynomial model. Based on the Jakes process, an auto-regressive (AR) model of the polynomial coefficients dynamics is built, making it possible to employ the Kalman filter estimator for the polynomial coefficients. Hence, the channel matrix can be easily computed. In order to perform polynomial coefficients estimation, we use the estimate along with the channel matrix output to recover the transmitted data. On can, in turn, use the detected data along with pilots to enhance the polynomial coefficients estimate giving rise to an iterative technique for complex gains and data recovery. The detection is performed over the ICI-free data symbol thanks to the use of a QR (orthogonal-triangle) decomposition [11] of the channel matrix, which is better compared to SIS equalizer. The present proposed algorithm has a good performance for very high Doppler spread $\left(f_{d} T>10 \%\right)$.

This paper is organized as follows: Section II introduces the OFDM system and the polynomial modeling. Section III describes the AR model for the polynomial coefficients and the Kalman filter. Section IV covers the algorithm for joint complex gains and data estimation. Section $\mathrm{V}$ presents the simulations results which validate our technique. Finally, our conclusions are presented in Section VI.

The notations adopted are as follows: Upper (lower) bold 
face letters denote matrices (column vectors). $[\mathbf{x}]_{k}$ denotes the $k$ th element of the vector $\mathbf{x}$, and $[\mathbf{X}]_{k, m}$ denotes the $[k, m]$ th element of the matrix $\mathbf{X}$. We will use the matlab notation $\mathbf{X}\left[k_{1}: k_{2}, m_{1}: m_{2}\right]$ to extract a submatrix within $\mathbf{X}$ from row $k_{1}$ to row $k_{2}$ and from column $m_{1}$ to column $m_{2} . \mathbf{I}_{N}$ is a $N \times N$ identity matrix and $\mathbf{0}_{N, L}$ is a $N \times L$ matrix of zeros $\left(\mathbf{0}_{N}=\mathbf{0}_{N, N}\right) \cdot \operatorname{diag}\{\mathbf{x}\}$ is a diagonal matrix with $\mathbf{x}$ on its main diagonal, $\operatorname{diag}\{\mathbf{X}\}$ is a vector whose elements are the elements of the main diagonal of $\mathbf{X}$ and $\operatorname{blkdiag}\{\mathbf{X}, \mathbf{Y}\}$ is a block diagonal matrix with the matrices $\mathbf{X}$ and $\mathbf{Y}$ on its main diagonal. The superscripts $(\cdot)^{T}$ and $(\cdot)^{H}$ stand respectively for transpose and Hermitian operators. $\operatorname{Tr}(\cdot)$ and $\mathrm{E}[\cdot]$ are the trace and expectation operations, respectively. $J_{0}(\cdot)$ is the zerothorder Bessel function of the first kind.

\section{OFDM System AND POLYNOMIAL MOdELING}

\section{A. OFDM System Model}

Consider an OFDM system with $\mathrm{N}$ sub-carriers, and a cyclic prefix length $N_{g}$. The duration of an OFDM symbol is $T=v T_{s}$, where $T_{s}$ is the sampling time and $v=N+N_{g}$. Let $\mathbf{x}_{(n)}=\left[x_{(n)}\left[-\frac{N}{2}\right], x_{(n)}\left[-\frac{N}{2}+1\right], \ldots, x_{(n)}\left[\frac{N}{2}-1\right]\right]^{T}$ be the $n$th transmitted OFDM symbol, where $\left\{x_{(n)}[b]\right\}$ are normalized QAM-symbols (i.e., $\mathrm{E}\left[x_{(n)}[b] x_{(n)}[b]^{*}\right]=1$ ). After transmission over a multi-path Rayleigh channel, the $n$th received OFDM symbol $\mathbf{y}_{(n)}=\left[y_{(n)}\left[-\frac{N}{2}\right], y_{(n)}\left[-\frac{N}{2}+1\right], \ldots, y_{(n)}\left[\frac{N}{2}-\right.\right.$ $1]]^{T}$ is given by [2] [1]:

$$
\mathbf{y}_{(n)}=\mathbf{H}_{(n)} \mathbf{x}_{(n)}+\mathbf{w}_{(n)}
$$

where $\mathbf{w}_{(n)}=\left[w_{(n)}\left[-\frac{N}{2}\right], w_{(n)}\left[-\frac{N}{2}+1\right], \ldots, w_{(n)}\left[\frac{N}{2}-1\right]\right]^{T}$ is a complex Gaussian noise vector with covariance matrix $\sigma^{2} \mathbf{I}_{N}$ and $\mathbf{H}_{(n)}$ is a $N \times N$ channel matrix with elements given by:

$$
\left[\mathbf{H}_{(n)}\right]_{k, m}=\frac{1}{N} \sum_{l=1}^{L}\left[e^{-j 2 \pi\left(\frac{m-1}{N}-\frac{1}{2}\right) \tau_{l}} \sum_{q=0}^{N-1} \alpha_{l}^{(n)}\left(q T_{s}\right) e^{j 2 \pi \frac{m-k}{N} q}\right]
$$

where $L$ is the number of paths, $\alpha_{l}$ is the $l$ th complex gain of variance $\sigma_{\alpha_{l}}^{2}$ and $\tau_{l} \times T_{s}$ is the $l$ th delay $\left(\tau_{l}\right.$ is not necessarily an integer, but $\tau_{L}<N_{g}$ ). The $\mathrm{L}$ individual elements of $\left\{\alpha_{l}^{(n)}\left(q T_{s}\right)=\alpha_{l}\left(q T_{s}+n T\right)\right\}$ are uncorrellated. They are wide-sense stationary (WSS), narrow-band complex Gaussian processes, with the so-called Jakes' power spectrum of maximum Doppler frequency $f_{d}$ (i.e., $\mathrm{E}\left[\alpha_{l}\left(q_{1} T_{s}\right) \alpha_{l}^{*}\left(q_{2} T_{s}\right)\right]=$ $\left.\sigma_{\alpha_{l}}^{2} J_{0}\left(2 \pi f_{d} T_{s}\left(q_{1}-q_{2}\right)\right)\right)$ [15]. The average energy of the channel is normalized to one, i.e., $\sum_{l=1}^{L} \sigma_{\alpha_{l}}^{2}=1$.

\section{B. Complex Gain Polynomial Modeling}

In [8], a piece-wise linear method is used to approximate the equivalent discrete-time channel taps. In [2], the authors show that the time-variation of Rayleigh channel complex gain, within $N_{c}$ OFDM symbols, can be approximated by a polynomial model of $N_{c}$ coefficients, choosen according to the Doppler spread $f_{d} T$.

In this section, in order to make the approximation in [2] more accurate for high Doppler spread, each Rayleigh channel complex gain $\boldsymbol{\alpha}_{l}^{(n)}=\left[\alpha_{l}^{(n)}\left(-N_{g} T_{s}\right), \ldots, \alpha_{l}^{(n)}\left((N-1) T_{s}\right)\right]^{T}$, within one OFDM symbol, is approximated by a polynomial model of $N_{c}$ coefficients (i.e., a $\left(N_{c}-1\right)$ degree polynomial). The optimal polynomial $\boldsymbol{\alpha}_{\mathbf{p o l}_{l}}^{(n)}$, which is least-squares fitted (linear and polynomial regression) [12] to $\boldsymbol{\alpha}_{l}^{(n)}$, and its $N_{c}$ coefficients $\mathbf{c}_{l}^{(n)}=\left[c_{1, l}^{(n)}, \ldots, c_{N_{c}, l}^{(n)}\right]^{T}$ are given by:

$\boldsymbol{\alpha}_{\mathbf{p o l}}^{(n)}=\mathbf{Q}^{T} \mathbf{c}_{l}^{(n)}=\mathbf{S} \boldsymbol{\alpha}_{l}^{(n)}$ and $\mathbf{c}_{l}^{(n)}=\left(\mathbf{Q} \mathbf{Q}^{T}\right)^{-1} \mathbf{Q} \boldsymbol{\alpha}_{l}^{(n)}$

where $\mathbf{Q}$ and $\mathbf{S}$ are a $N_{c} \times v$ and a $v \times v$ matrices, respectively, defined as:

$$
\begin{aligned}
{[\mathbf{Q}]_{k, m} } & =\left(m-N_{g}-1\right)^{(k-1)} \\
\mathbf{S} & =\mathbf{Q}^{T}\left(\mathbf{Q} \mathbf{Q}^{T}\right)^{-1} \mathbf{Q}
\end{aligned}
$$

It provides the MMSE approximation for all polynomials containing $N_{c}$ coefficients, given by:

$$
\operatorname{MMSE}_{l}=\frac{1}{v} \mathrm{E}\left[\boldsymbol{\xi}_{l}^{(n)^{H}} \boldsymbol{\xi}_{l}^{(n)}\right]=\frac{1}{v} \operatorname{Tr}\left(\left(\mathbf{I}_{v}-\mathbf{S}\right) \mathbf{R}_{\boldsymbol{\alpha}_{l}}^{(0)}\left(\mathbf{I}_{v}-\mathbf{S}^{T}\right)\right)
$$

where $\boldsymbol{\xi}_{l}^{(n)}=\boldsymbol{\alpha}_{l}^{(n)}-\boldsymbol{\alpha}_{\mathbf{p o l} l_{l}}^{(n)}$ is the model error and $\mathbf{R}_{\boldsymbol{\alpha}_{l}}^{(s)}=$ $\mathrm{E}\left[\boldsymbol{\alpha}_{l}^{(n)} \boldsymbol{\alpha}_{l}^{(n-s)^{H}}\right]$ is the $v \times v$ correlation matrix of $\boldsymbol{\alpha}_{l}^{(n)}$ with elements given by:

$$
\left[\mathbf{R}_{\boldsymbol{\alpha}_{l}}^{(s)}\right]_{k, m}=\sigma_{\alpha_{l}}^{2} J_{0}\left(2 \pi f_{d} T_{s}(k-m+s v)\right)
$$

Under this polynomial approximation, the observation model in (1) for the $n$th OFDM symbol can be rewritten as:

$$
\mathbf{y}_{(n)}=\mathcal{K}_{(n)} \mathbf{c}_{(n)}+\mathbf{w}_{(n)}
$$

where $\mathbf{c}_{(n)}=\left[\mathbf{c}_{1}^{(n)^{T}}, \ldots, \mathbf{c}_{L}^{(n)^{T}}\right]^{T}$ is a $L N_{c} \times 1$ vector, $\mathcal{K}_{(n)}=\frac{1}{N}\left[\mathbf{Z}_{1}^{(n)}, \ldots, \mathbf{Z}_{L}^{(n)}\right]$ is a $N \times L N_{c}$ matrix and $\mathbf{Z}_{l}^{(n)}=$ $\left[\mathbf{M}_{1} \operatorname{diag}\left\{\mathbf{x}_{(n)}\right\} \mathbf{f}_{l}, \ldots, \mathbf{M}_{N_{c}} \operatorname{diag}\left\{\mathbf{x}_{(n)}\right\} \mathbf{f}_{l}\right]$ is a $N \times N_{c}$ matrix, where $\mathbf{f}_{l}$ is the $l$ th column of the $N \times L$ Fourier matrix $\mathbf{F}$ and $\mathbf{M}_{d}$ is a $N \times N$ matrix given by:

$$
[\mathbf{F}]_{k, l}=e^{-j 2 \pi\left(\frac{k-1}{N}-\frac{1}{2}\right) \tau_{l}} \text { and }\left[\mathbf{M}_{d}\right]_{k, m}=\sum_{q=0}^{N-1} q^{d-1} e^{j 2 \pi \frac{m-k}{N} q}
$$

Moreover, the channel matrix can be easily computed as [2]:

$$
\mathbf{H}_{(n)}=\sum_{d=1}^{N_{c}} \mathbf{M}_{d} \operatorname{diag}\left\{\mathbf{F} \chi_{d}^{(n)}\right\}
$$

where $\chi_{d}^{(n)}=\left[c_{d, 1}^{(n)}, \ldots, c_{d, L}^{(n)}\right]^{T}$. The matrices $\mathbf{M}_{d}$ can be easily computed and stored, using the properties of power series.

\section{AR MODEL AND KALMAN FILTER}

\section{A. The AR Model for $\boldsymbol{c}^{(n)}$}

$\mathbf{c}_{l}^{(n)}$ are correlated complex Gaussian variables with zeromeans and correlation matrix given by:

$\mathbf{R}_{\mathbf{c}_{l}}^{(s)}=\mathrm{E}\left[\mathbf{c}_{l}^{(n)} \mathbf{c}_{l}^{(n-s)^{H}}\right]=\left(\mathbf{Q} \mathbf{Q}^{T}\right)^{-1} \mathbf{Q} \mathbf{R}_{\boldsymbol{\alpha}_{l}}^{(s)} \mathbf{Q}^{T}\left(\mathbf{Q} \mathbf{Q}^{T}\right)^{-1}$

Hence, the dynamics of $\mathbf{c}_{l}^{(n)}$ can be well modeled by an autoregressive (AR) process [13] [14]. A complex AR process of order $p$ can be generated as:

$$
\mathbf{c}_{l}^{(n)}=-\sum_{i=1}^{p} \mathbf{A}_{l}^{(i)} \mathbf{c}_{l}^{(n-i)}+\mathbf{u}_{l}^{(n)}
$$


where $\mathbf{A}_{l}^{(1)}, \ldots, \mathbf{A}_{l}^{(p)}$ are $N_{c} \times N_{c}$ matrices and $\mathbf{u}_{l}^{(n)}$ is a $N_{c} \times 1$ complex Gaussian vector with covariance matrix $\mathbf{U}_{l}$. $\mathbf{A}_{l}^{(1)}, \ldots, \mathbf{A}_{l}^{(p)}$ and $\mathbf{U}_{l}$ are the AR model parameters obtained by solving the set of Yule-Walker equations defined as:

$$
\mathbf{T}_{l} \mathbf{A}_{l}=-\mathbf{V}_{l} \quad \text { and } \quad \mathbf{U}_{l}=\mathbf{R}_{\mathbf{c}_{l}}^{(0)}+\sum_{i=1}^{p} \mathbf{A}_{l}^{(i)} \mathbf{R}_{\mathbf{c}_{l}}^{(-i)}
$$

where $\mathbf{A}_{l}=\left[\mathbf{A}_{l}^{(1)^{T}}, \ldots, \mathbf{A}_{l}^{(p)^{T}}\right]^{T}, \mathbf{V}_{l}=\left[\mathbf{R}_{\mathbf{c}_{l}}^{(1)^{T}}, \ldots, \mathbf{R}_{\mathbf{c}_{l}}^{(p)^{T}}\right]^{T}$ are $p N_{c} \times N_{c}$ matrices and $\mathbf{T}_{l}$ is a $p N_{c} \times p N_{c}$ correlation matrix defined by:

$$
\mathbf{T}_{l}=\left[\begin{array}{ccc}
\mathbf{R}_{\mathbf{c}_{l}}^{(0)} & \cdots & \mathbf{R}_{\mathbf{c}_{l}}^{(-p+1)} \\
\vdots & \ddots & \vdots \\
\mathbf{R}_{\mathbf{c}_{l}}^{(p-1)} & \cdots & \mathbf{R}_{\mathbf{c}_{l}}^{(0)}
\end{array}\right]
$$

Using (12), we obtain the AR model of order $p$ for $\mathbf{c}_{(n)}$ :

$$
\mathbf{c}_{(n)}=-\sum_{i=1}^{p} \mathbf{A}_{(i)} \mathbf{c}_{(n-i)}+\mathbf{u}_{(n)}
$$

where $\mathbf{A}_{(i)}=$ blkdiag $\left\{\mathbf{A}_{1}^{(i)}, \ldots, \mathbf{A}_{L}^{(i)}\right\}$ is a $L N_{c} \times L N_{c}$ matrix and $\mathbf{u}_{(n)}=\left[\mathbf{u}_{1}^{(n)^{T}}, \ldots, \mathbf{u}_{L}^{(n)^{T}}\right]^{T}$ is a $L N_{c} \times 1$ complex Gaussian vector with covariance matrix $\mathbf{U}=\operatorname{blkdiag}\left\{\mathbf{U}_{1}, \ldots, \mathbf{U}_{L}\right\}$.

\section{B. The Kalman Filter}

Based on the AR model of $\mathbf{c}_{(n)}$ in (15), we define the state space model for the OFDM system as $\mathbf{g}_{(n)}=$ $\left[\mathbf{c}_{(n)}^{T}, \ldots, \mathbf{c}_{(n-p+1)}^{T}\right]^{T}$. Thus, using (15) and (8), we obtain:

$$
\begin{aligned}
& \mathbf{g}_{(n)}=\mathbf{S}_{1} \mathbf{g}_{(n-1)}+\mathbf{S}_{2} \mathbf{u}_{(n)} \\
& \mathbf{y}_{(n)}=\mathbf{S}_{3} \mathbf{g}_{(n)}+\mathbf{w}_{(n)}
\end{aligned}
$$

where $\mathbf{S}_{2}=\left[\mathbf{I}_{L N_{c}}, \mathbf{0}_{L N_{c},(p-1) L N_{c}}\right]^{T}$ is a $p L N_{c} \times L N_{c}$ matrix, $\mathbf{S}_{3}=\left[\mathcal{K}_{(n)}, \mathbf{0}_{N,(p-1) L N_{c}}\right]$ is a $N \times p L N_{c}$ matrix and $\mathbf{S}_{1}$ is a $p L N_{c} \times p L N_{c}$ matrix defined as:

$$
\mathbf{S}_{1}=\left[\begin{array}{ccccc}
-\mathbf{A}_{(1)} & -\mathbf{A}_{(2)} & -\mathbf{A}_{(3)} & \ldots & -\mathbf{A}_{(p)} \\
\mathbf{I}_{L N_{c}} & \mathbf{0}_{L N_{c}} & \mathbf{0}_{L N_{c}} & \ldots & \mathbf{0}_{L N_{c}} \\
\mathbf{0}_{L N_{c}} & \mathbf{I}_{L N_{c}} & \mathbf{0}_{L N_{c}} & \ldots & \mathbf{0}_{L N_{c}} \\
\vdots & \ddots & \ddots & \ddots & \vdots \\
\mathbf{0}_{L N_{c}} & \cdots & \mathbf{0}_{L N_{c}} & \mathbf{I}_{L N_{c}} & \mathbf{0}_{L N_{c}}
\end{array}\right]
$$

The state model (16) and the observation model (17) allow us to use Kalman filter to adaptively track the polynomial coefficients $\mathbf{c}_{(n)}$. Let $\hat{\mathbf{g}}_{(n)}$ be our a priori state estimate at step $n$ given knowledge of the process prior to step $n, \hat{\mathbf{g}}_{(n \mid n)}$ be our a posteriori state estimate at step $n$ given measurement $\mathbf{y}_{(n)}$ and, $\mathbf{P}_{(n)}$ and $\mathbf{P}_{(n \mid n)}$ are the a priori and the a posteriori error estimate covariance matrix of size $p L N_{c} \times p L N_{c}$, respectively. We initialize the Kalman filter with $\mathbf{g}_{(0 \mid 0)}=\mathbf{0}_{p L N_{c}, 1}$ and $\mathbf{P}_{(0 \mid 0)}$ given by:

$$
\mathbf{P}_{(0 \mid 0)\left[t(l, s), t\left(l, s^{\prime}\right)\right]}=\mathbf{R}_{\mathbf{c}_{l}}^{\left(s^{\prime}-s\right)} \text { for } l \in[1, L] s, s^{\prime} \in[0, p-1]
$$

where $t(l, s)=1+(l-1) N_{c}+s L N_{c}: l N_{c}+s L N_{c}$ and $\mathbf{R}_{\mathbf{c}_{l}}^{(s)}$ is the correlation matrix of $\mathbf{c}_{l}{ }^{(n)}$ defined in (11). Notice that there are zero matrices between the block matrices $\mathbf{R}_{\mathbf{c}_{l}}^{(s)}$ since the L complex gains are uncorrellated with respect to each other. For $K=L=2, \mathbf{P}^{(0 \mid 0)}$ is given by:

$$
\mathbf{P}_{(0 \mid 0)}=\left[\begin{array}{cccc}
\mathbf{R}_{\mathbf{c}_{1}}^{(0)} & \mathbf{0}_{N_{c}} & \mathbf{R}_{\mathbf{c}_{1}}^{(1)} & \mathbf{0}_{N_{c}} \\
\mathbf{0}_{N_{c}} & \mathbf{R}_{\mathbf{c}_{2}}^{(0)} & \mathbf{0}_{N_{c}} & \mathbf{R}_{\mathbf{c}_{2}}^{(1)} \\
\mathbf{R}_{\mathbf{c}_{1}}^{(-1)} & \mathbf{0}_{N_{c}} & \mathbf{R}_{\mathbf{c}_{1}}^{(0)} & \mathbf{0}_{N_{c}} \\
\mathbf{0}_{N_{c}} & \mathbf{R}_{\mathbf{c}_{2}}^{(-1)} & \mathbf{0}_{N_{c}} & \mathbf{R}_{\mathbf{c}_{2}}^{(0)}
\end{array}\right]
$$

The Kalman filter is a recursive algorithm composed of two stages: Time Update Equations and Measurement Update Equations. These two stages are defined as:

Time Update Equations:

$$
\begin{aligned}
\hat{\mathbf{g}}_{(n)} & =\mathbf{S}_{1} \hat{\mathbf{g}}_{(n-1 \mid n-1)} \\
\mathbf{P}_{(n)} & =\mathbf{S}_{1} \mathbf{P}_{(n-1 \mid n-1)} \mathbf{S}_{1}^{H}+\mathbf{S}_{2} \mathbf{U} \mathbf{S}_{2}^{H}
\end{aligned}
$$

\section{Measurement Update Equations:}

$$
\begin{aligned}
\mathbf{K}_{(n)} & =\mathbf{P}_{(n)} \mathbf{S}_{3}^{H}\left(\mathbf{S}_{3} \mathbf{P}_{(n)} \mathbf{S}_{3}^{H}+\sigma^{2} \mathbf{I}_{N}\right)^{-1} \\
\hat{\mathbf{g}}_{(n \mid n)} & =\hat{\mathbf{g}}_{(n)}+\mathbf{K}_{(n)}\left(\mathbf{y}_{(n)}-\mathbf{S}_{3} \hat{\mathbf{g}}_{(n)}\right) \\
\mathbf{P}_{(n \mid n)} & =\mathbf{P}_{(n)}-\mathbf{K}_{(n)} \mathbf{S}_{3} \mathbf{P}_{(n)}
\end{aligned}
$$

where $\mathbf{K}_{(n)}$ is the Kalman gain. The Time Update Equations are responsible for projecting forward (in time) the current state and error covariance estimates to obtain the a priori estimates for the next time step. The Measurement Update Equations are responsible for the feedback, i.e., for incorporating a new measurement into the a priori estimate to obtain an improved a posteriori estimate. The Time Update Equations can also be thought of a predictor equations, while the Measurement Update Equations can be thought of a corrector equations.

\section{JOINT QR-DETECTION AND KALMAN ESTIMATION}

\section{A. Data QR-detection}

The QR-detection allow us to estimate the data symbol with free ICI. First, we transform the channel matrix $\mathbf{H}_{(n)}$ by performing a so-called QR-decomposition:

$$
\mathbf{H}_{(n)}=\mathcal{Q}_{(n)} \mathcal{R}_{(n)}
$$

where $\mathcal{Q}_{(n)}$ is a $N \times N$ unitary matrix (i.e., $\mathcal{Q}_{(n)}^{H} \mathcal{Q}_{(n)}=\mathbf{I}_{N}$ ) and $\mathcal{R}_{(n)}$ is a $N \times N$ upper triangular matrix. Then, we can rewrite equation (1) as:

$$
\mathbf{y}_{(n)}^{\prime}=\mathcal{Q}_{(n)}^{H} \mathbf{y}_{(n)}=\mathcal{R}_{(n)} \mathbf{x}_{(n)}+\mathcal{Q}_{(n)}^{H} \mathbf{w}_{(n)}
$$

The upper triangular form of $\boldsymbol{\mathcal { R }}_{(n)}$ now allow us to iteratively calculate estimates, with free ICI, for the originally data symbols $\left\{\left[\mathbf{x}_{(n)}\right]_{N},\left[\mathbf{x}_{(n)}\right]_{N-1}, \ldots,\left[\mathbf{x}_{(n)}\right]_{1}\right\}$ as:

$$
\begin{aligned}
{\left[\tilde{\mathbf{x}}_{(n)}\right]_{k} } & =\frac{\left[\mathbf{y}_{(n)}^{\prime}\right]_{k}-\sum_{m=k+1}^{N}\left[\boldsymbol{\mathcal { R }}_{(n)}\right]_{k, m}\left[\hat{\mathbf{x}}_{(n)}\right]_{m}}{\left[\boldsymbol{\mathcal { R }}_{(n)}\right]_{k, k}} \\
{\left[\hat{\mathbf{x}}_{(n)}\right]_{k} } & =\mathcal{O}\left(\left[\tilde{\mathbf{x}}_{(n)}\right]_{k}\right)
\end{aligned}
$$

where $\mathcal{O}($.$) denotes the quantization operation appropriate to$ the constellation in use. 


\section{B. Iterative Algorithm}

In the iterative algorithm for joint data QR-detection and complex gains Kalman estimation, the $N_{p}$ pilots subcarriers are evently inserted into the $\mathrm{N}$ subcarriers at the positions $\mathcal{P}=\left\{p_{r} \mid p_{r}=(r-1) L_{f}+1, r=1, \ldots, N_{p}\right\}$, where $L_{f}$ is the distance between two adjacent pilots. The algorithm proceeds as follows:

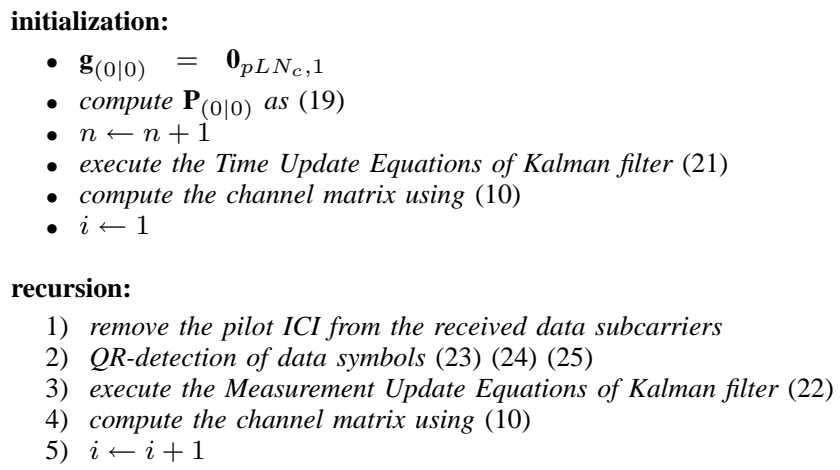

1) remove the pilot ICI from the received data subcarriers

2) QR-detection of data symbols (23) (24) (25)

3) execute the Measurement Update Equations of Kalman filter (22)

4) compute the channel matrix using (10)

5) $i \leftarrow i+1$

where $i$ represents the iteration number.

\section{Mean Square Error (MSE) Analysis}

The error between the $l$ th exact complex gain and the $l$ th estimated polynomial $\hat{\boldsymbol{\alpha}}_{\mathbf{p o l}_{l}}^{(n)}$ is given by:

$$
\mathbf{e}_{l}^{(n)}=\boldsymbol{\alpha}_{l}^{(n)}-\hat{\boldsymbol{\alpha}}_{\mathbf{p o l}_{l}}^{(n)}=\boldsymbol{\xi}_{l}^{(n)}+\mathbf{Q}^{T} \mathbf{e}_{\mathbf{c}_{l}}^{(n)}
$$

where $\mathbf{e}_{\mathbf{c}_{l}}^{(n)}=\mathbf{c}_{l}^{(n)}-\hat{\mathbf{c}}_{l}^{(n)}$ and $\boldsymbol{\xi}_{l}^{(n)}$ is the polynomial model error defined in section II-B. Neglecting the cross-covariance terms between $\boldsymbol{\xi}_{l}^{(n)}$ and $\mathbf{e}_{\mathbf{c}_{l}}^{(n)}$, the mean square error (MSE) between $\boldsymbol{\alpha}_{l}^{(n)}$ and $\boldsymbol{\alpha}_{\mathbf{p o l}}^{(n)}$ is given by:

$$
\begin{aligned}
\operatorname{MSE}_{l} & =\frac{1}{v} \mathrm{E}\left[\mathbf{e}_{l}^{(n)^{H}} \mathbf{e}_{l}^{(n)}\right] \\
& =\mathrm{MMSE}_{l}+\frac{1}{v} \operatorname{Tr}\left(\mathbf{Q}^{T} \mathbf{M S E} \mathbf{c}_{l} \mathbf{Q}\right)
\end{aligned}
$$

where $\mathbf{M S E}_{\mathbf{c}_{l}}=\mathrm{E}\left[\mathbf{e}_{\mathbf{c}_{l}}^{(n)} \mathbf{e}_{\mathbf{c}_{l}}^{(n)}{ }^{H}\right]$. Notice that, at the convergence of the Kalman filter, we have:

$$
\mathbf{M S E}_{\mathbf{c}_{l}}=\mathbf{P}_{(n \mid n)}[t(l, 0), t(l, 0)]
$$

provided that the data symbols are perfectly estimated (i.e., data-aided).

The on-line Bayesian Cramer-Rao Bound (BCRB) is an important criterion for evaluting the quality of our complex gains Kalman estimation. The on-line BCRB for the estimation of $\boldsymbol{\alpha}_{l}^{(n)}$, in data-aided (DA) context, is studied in [3]:

$$
\operatorname{BCRB}\left(\boldsymbol{\alpha}_{l}^{(\infty)}\right)=\operatorname{MMSE}_{l}+\frac{1}{v} \operatorname{Tr}\left(\mathbf{Q}^{T} \mathbf{B C R B}\left(\mathbf{c}_{l}^{(\infty)}\right) \mathbf{Q}\right)
$$

where $\mathbf{B C R B}\left(\mathbf{c}_{l}^{(K)}\right)$ is the on-line BCRB associated to the estimation of $\mathbf{c}_{l}^{(K)}$ which is given by:

$$
\operatorname{BCRB}\left(\mathbf{c}_{l}^{(K)}\right)=\operatorname{BCRB}(\mathbf{c})_{[t(l, 0), t(l, 0)]}
$$

$\operatorname{BCRB}(\mathbf{c})$ is the on-line BCRB for the estimation of $\mathbf{c}=$ $\left[\mathbf{c}_{(K)}{ }^{T}, \ldots, \mathbf{c}_{(1)}{ }^{T}\right]^{T}$ in DA context which is given by:

$\operatorname{BCRB}(\mathbf{c})=\left(\operatorname{blkdiag}\left\{\mathbf{J}_{(K)}, \ldots, \mathbf{J}_{(2)}, \mathbf{J}_{(1)}\right\}+\mathbf{R}_{\mathbf{c}}^{-1}\right)^{-1}$

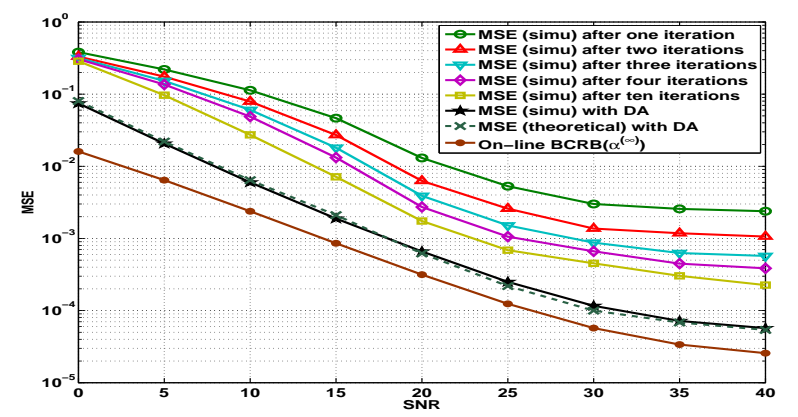

Fig. 1. MSE vs SNR for $f_{d} T=0.3$ and $N_{c}=3$
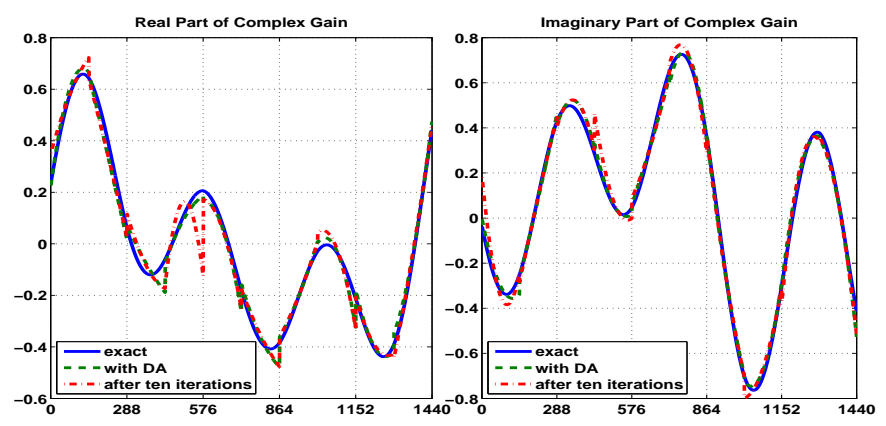

Fig. 2. The Kalman estimated complex gain of the 2nd path over 10 OFDM symbols after ten iterations for $\mathrm{SNR}=20 d B, f_{d} T=0.3$ and $N_{c}=3$

where $\mathbf{R}_{\mathbf{c}}$ is calculated in the same way as $\mathbf{P}_{(0 \mid 0)}$ with $s, s^{\prime} \in$ $[0, K-1]$, and $\mathbf{J}_{(n)}=\frac{1}{N^{2} \sigma^{2}} \mathcal{F}_{(n)}^{H} \mathcal{M} \mathcal{F}_{(n)} . \mathcal{M}$ and $\mathcal{F}_{(n)}$ are a $N N_{c} \times N N_{c}$ and a $N N_{c} \times L N_{c}$ matrices, respectively, defined as:

$$
\begin{aligned}
\mathcal{M} & =\left[\begin{array}{ccc}
\mathcal{M}_{1,1} & \cdots & \mathcal{M}_{1, N_{c}} \\
\vdots & \ddots & \vdots \\
\mathcal{M}_{N_{c}, 1} & \cdots & \mathcal{M}_{N_{c}, N_{c}}
\end{array}\right] \\
\mathcal{F}_{(n)} & =\left[\begin{array}{lll}
\mathcal{F}_{1}^{(n)} & \cdots & \mathcal{F}_{L}^{(n)}
\end{array}\right]
\end{aligned}
$$

where $\mathcal{M}_{d, d^{\prime}}$ and $\mathcal{F}_{l}^{(n)}$ are a $N \times N$ and a $N N_{c} \times N_{c}$ matrices, respectively, defined as:

$$
\begin{aligned}
\mathcal{M}_{d, d^{\prime}} & =\operatorname{diag}\left\{\operatorname{diag}\left\{\mathbf{M}_{d}^{H} \mathbf{M}_{d^{\prime}}\right\}\right\} \\
\mathcal{F}_{l}^{(n)} & =\operatorname{blkdiag}\left\{\mathbf{v}_{l}^{(n)}, \mathbf{v}_{l}^{(n)}, \ldots, \mathbf{v}_{l}^{(n)}\right\}
\end{aligned}
$$

with $\mathbf{v}_{l}^{(n)}=\operatorname{diag}\left\{\mathbf{x}_{(n)}\right\} \mathbf{f}_{l}$. It should be noted that, when the number of observations $K$ increases, $\mathbf{B C R B}\left(\mathbf{c}_{l}^{(K)}\right)$ decreases and converges to an asymptote $\mathbf{B C R B}\left(\mathbf{c}_{l}^{(\infty)}\right)$.

\section{Simulation}

In this section, we verify the theory by simulation and we test the performance of the iterative algorithm. The normalized channel model is GSM Rayleigh model [2] [1] with $L=6$ paths and maximum delay $\tau_{\max }=10 T_{s}$. A 4QAM-OFDM system with normalized symbols, $N=128$ subcarriers, $N_{g}=\frac{N}{8}$ subcarriers, $N_{p}=32$ pilots (i.e., $\left.L_{f}=4\right)$ and $\frac{1}{T_{s}}=2 M H z$ is used (note that $(S N R) d B=\left(\frac{E_{b}}{N_{0}}\right) d B+3 d B$ ). These parameters are selected in order to be in concordance with the standard Wimax IEEE802.16e. The MSE and the BER are evaluated under a rapid time-varying channel such as $f_{d} T=0.1, f_{d} T=0.2$ and $f_{d} T=0.3$ corresponding to a vehicle speed $V_{m}=140 \mathrm{~km} / \mathrm{h}, V_{m}=280 \mathrm{~km} / \mathrm{h}$ and 


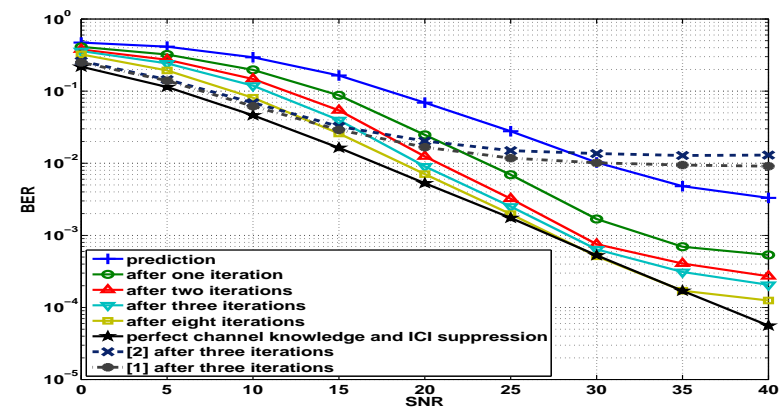

Fig. 3. BER vs SNR for $N_{c}=3$ and $f_{d} T=0.2$

$V_{m}=420 \mathrm{~km} / \mathrm{h}$, respectively, for $f_{c}=10 \mathrm{GHz}$. In order to decrease the complexity of the Kalman filter, we choose an AR model of order $p=1$.

Fig. 1 shows the evolution of $M S E$ versus SNR, with the iterations, for $f_{d} T=0.3$ and $N_{c}=3$. It is observed that, with DA, the $M S E$ obtained by simulation agrees with the theoretical value of $M S E$ given by (28). Fig. 1 also shows that $M S E$ with DA is very close to the on-line BCRB. This means that the Kalman filter works very well. After four and ten iterations, a great improvement is realized and the MSE is close to the MSE with DA. For illustration, Fig. 2 gives the real and the imaginary parts of the exact, the DA estimated and the estimated (with pilots after ten iterations) complex gain of the second path. This is obtained for one channel realization over 10 OFDM symbols with $\mathrm{SNR}=20 d B, f_{d} T=0.3$ and $N_{c}=3$. We notice how good is the estimation of multi-path complex gains for very rapidly channels.

Fig. 3 gives the BER performance of our algorithm for $f_{d} T=0.2$ with $N_{c}=3$, compared to the algorithms in [2] and [1]. As reference, we plotted the performance obtained with perfect knowledge of channel and perfect suppression of ICI. This result shows that our algorithm performs better than the algorithms proposed in [2] and [1]. After eight iterations, a significant improvement occurs; the performance of our algorithm and the performance obtained with perfect knowledge of channel and ICI are very close. At a very high $\mathrm{SNR}$, it is normal to not reach the reference because we have a small error floor due to the data symbol detection error.

Fig. 4 gives the BER performance after ten iterations of our iterative algorithm, for $N_{c}=3$ and $f_{d} T=0.3$, with imperfect delay knowledge. SD denotes the standard deviation of the time delay errors (modeled as zero mean Gaussian variables). It can be noticed that the algorithm is not very sensitive to a delay error of $\mathrm{SD}<0.1 T_{s}$. By using the ESPRIT method [9] to estimate the delays, we have a $\mathrm{SD}<0.05 T_{s}$, for all SNR as shown in Fig. 5. When combined with the ESPRIT method, our algorithm thus has negligible sensitivity to delay errors.

\section{CONCLUSION}

In this paper, we have presented a new iterative algorithm for joint multi-path Rayleigh channel complex gains and data recovery in fast fading environments. The rapid time-variation complex gain within one OFDM symbole are approximated by a polynomial model. Exploiting the fact that the delays can be assumed to be invariant (over several symbols) and perfectly estimated, the polynomial coefficients are tracked using the

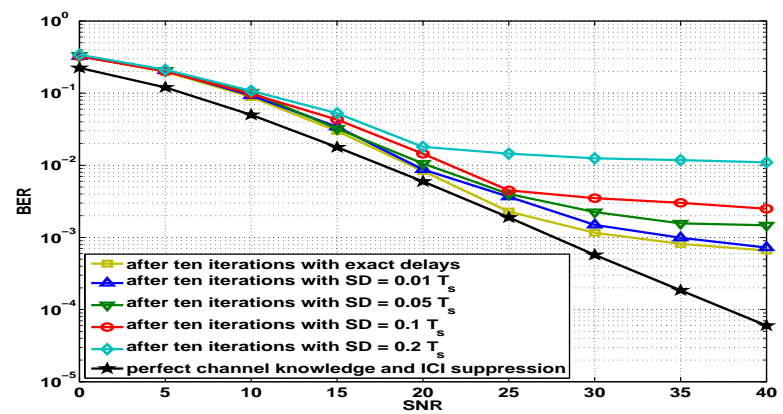

Fig. 4. Comparison of BER, for the case of imperfect knowledge of delays, with $N_{c}=3$ and $f_{d} T=0.3$

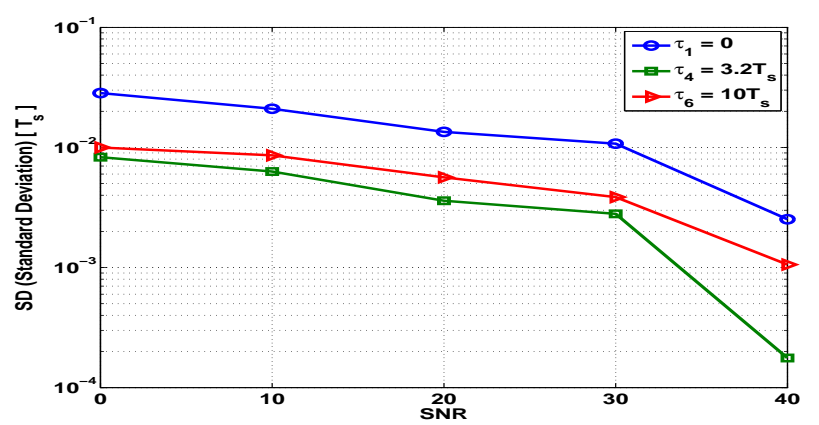

Fig. 5. Delay estimation errors for the first, fourth and sixth paths, using the ESPRIT method [9] (estimated correlation matrix, averaged over 1000 OFDM symbols, i.e $0.072 \mathrm{sec}$ ), for $f_{d} T=0.3$

Kalman filter. The data symbols are estimated by performing a QR-decomposition of the channel matrix. Theoretical analysis and simulation results show that our algorithm has a good performance for high Doppler spread.

\section{REFERENCES}

[1] H. Hijazi and L. Ros, “ Time-varying channel complex gains estimation and ICI suppression in OFDM systems" in IEEE GLOBAL COMMUNICATIONS Conf., Washington, USA, Nov. 2007.

[2] H. Hijazi and L. Ros, "Polynomial estimation of time-varying multi-path gains with intercarrier interference mitigation in OFDM systems" in IEEE Trans. Vehic. Techno., (To be appeared)

[3] H. Hijazi and L. Ros, “ Analytical Analysis of Bayesian Cramer-Rao Bound for Dynamical Rayleigh Channel Complex Gains Estimation in OFDM System " submitted to IEEE Trans. Signal Process.

[4] A. R. S. Bahai and B. R. Saltzberg, Multi-Carrier Dications: Theory and Applications of OFDM: Kluwer Academic/Plenum, 1999.

[5] M. Hsieh and C. Wei, "Channel estimation for OFDM systems based on comb-type pilot arrangement in frequency selective fading channels" in IEEE Trans. Consumer Electron., vol.44, no. 1, Feb. 1998.

[6] Z Tang, R. C. Cannizzaro, G. Leus and P. Banelli, "Pilot-assisted time-varying channel estimation for OFDM systems" in IEEE Trans. Signal Process., vol. 55, pp. 2226-2238, May 2007.

[7] S. Tomasin, A. Gorokhov, H. Yang and J.-P. Linnartz, "Iterative interference cancellation and channel estimation for mobile OFDM" in IEEE Trans. Wireless Commun., vol. 4, no. 1, pp. 238-245, Jan. 2005.

[8] Y. Mostofi and D. Cox, "ICI mitigation for pilot-aided OFDM mobile systems" in IEEE Trans. Wireless Commun., vol. 4, no. 12, pp. 765-774, March 2005.

[9] B. Yang, K. B. Letaief, R. S. Cheng and Z. Cao, "Channel estimation for OFDM transmisson in mutipath fading channels based on parametric channel modeling" in IEEE Trans. Commun., vol. 49, no. 3, pp. 467-479, March 2001.

[10] E. Simon, L. Ros and K. Raoof," Synchronization over rapidly time-varying multipath channel for CDMA downlink RAKE receivers in Time-Division mode", in IEEE Trans. Vehic. Techno., vol. 56. no. 4, Jul. 2007

[11] E. Anderson and Z. Bai, LAPACK User's Guide: Third Edition,SIAM, Philadelphia, 1999.

[12] Wikipedia contributors, "Linear regression", Wikipedia, The Free Encyclopedia.

[13] K. E. Baddour and N. C. Beaulieu, "Autoregressive modeling for fading channel simulation" in IEEE Trans. Wireless Commun., vol. 4, no. 4, pp. 1650-1662, July 2005.

[14] B. Anderson and J. B. Moore, Optimal filtering, Prentice-Hall, 1979.

[15] W. C. Jakes, Microwave Mobile Communications. Piscataway, NJ: IEEE Press, 1983. 